\title{
Prevalência de dengue clássica e dengue hemorrágica no Brasil, entre 2011 e 2015
}

\author{
Prevalence of dengue fever and dengue hemorrhagic fever, in Brazil, between 2011 and \\ 2015
}

Prevalencia de dengue clásico y dengue hemorrágico, en Brasil, entre 2011 y 2015

Tercio Cirqueira Correia ${ }^{1}$, Victor de Oliveira Flausino ${ }^{1}$, Leonardo Lins Figueiredo ${ }^{1}$, Thiago Vinícius dos Santos Ferreira', Tarcísio Veloso Rabelo', Tomaz Dario Fernandez Coelho', Anna Cecília Castro e Abreu ${ }^{1 *}$, Karina Andrade de Prince'.

\section{RESUMO}

Objetivo: Investigar a prevalência de internações por dengue clássica e dengue hemorrágica, no Brasil, entre 2011 e 2015. Métodos: Trata-se de estudo epidemiológico transversal, descritivo, de abordagem quantitativa, com dados obtidos por meio de banco de dados gerenciado pelo DATASUS. Foram 304.444 casos de internação por dengue e dengue hemorrágica no período estudado. Resultados: Para o período do estudo, o ano de maior número de casos para ambas as formas de dengue foi 2011, observa-se um maior predomínio do sexo feminino $(53,60 \%)$, a faixa etária mais acometida é de 20 a 39 anos $(31,3 \%)$, a cor/raça mais declarada é a parda, com $42,7 \%$. A região Nordeste apresentou o maior número absoluto para ambas as formas de dengue com 128.949 casos. A região Sudeste possui a maior proporção de casos de Dengue hemorrágica (4,54\%). Já a menor incidência em casos de ambas as formas de dengue encontra-se na região Sul, com apenas 10.731. O número de óbitos para cada 1.000 casos no período estudado mostra o ano de 2015 com a maior taxa de óbitos $(5,81 \%)$. Conclusão: $O$ estudo permitiu constatar as desigualdades regionais nas políticas de saúde pública como: controle do vetor, eficácia na notificação e equipe de saúde capacitada para $o$ atendimento.

Descritores: Dengue, Morbimortalidade, Óbitos, DATASUS.

\begin{abstract}
Objective: To investigate the prevalence of hospitalizations for dengue fever and dengue hemorrhagic fever, in Brazil, between 2011 and 2015. Methods: This was a cross-sectional epidemiological study, descriptive, quantitative approach, with data obtained by means of the database managed by DATASUS. Were 304.444 cases of hospitalization due to dengue fever and dengue hemorrhagic fever in the period studied. Results: For the study period, the year of the largest number of cases for both forms of dengue was 2011, there is a greater predominance of females $(53.60 \%)$, the most affected age is 20 to 39 years $(31.3 \%)$, the color/race more declared is mixed, with $42.7 \%$. The northeast region showed the highest absolute number for both forms of dengue fever with 128.949 cases. The Southeast region has the highest proportion of cases of dengue hemorrhagic fever (4.54\%). Already the lowest incidence in cases of both forms of dengue is located in the Southern Region, with only 10,731. The number of deaths for every 1,000 cases during the study period shows the year of 2015 with the highest rate of deaths $(5.81 \%)$. Conclusions: The study revealed the regional inequalities in public health policies as: vector control, efficiency in the notification and the health care team qualified for the service.
\end{abstract}

Key words: Dengue, Morbidity and mortality, Deaths, DATASUS.

${ }^{1}$ Faculdades Integradas Pitágoras- Montes Claros-MG. *E-mail: anna.cecilia.castro@gmail.com

SUBMETIDO EM: 10/2018

ACEITO EM: 11/2018

PUBLICADO EM: 4/2019

REAS/EJCH | Vol. Sup. 22 | e753 | DOI: https://doi.org/10.25248/reas.e753.2019 Página 1 de 8 


\section{RESUMEN}

Objectivo: Investigar la prevalencia de internaciones por dengue clásico y dengue hemorrágico en Brasil entre 2011 y 2015. Métodos: Se trata de un estudio epidemiológico transversal, descriptivo, de abordaje cuantitativo, con datos obtenidos a través de base de datos gestionado por DATASUS. Fueron 304.444 casos de internación por dengue y dengue hemorrágico en el período estudiado. Resultados: Para el período del estudio, el año de mayor número de casos para ambas formas de dengue fue 2011, se observa un mayor predominio del sexo femenino (53,60\%), el grupo de edad más acometido es de 20 a 39 (31,3\%), el color / raza más declarada es la parda, con el $42,7 \%$. La región Nordeste presentó el mayor número absoluto para ambas formas de dengue con 128.949 casos. La región Sudeste posee la mayor proporción de casos de Dengue hemorrágico (4,54\%). La menor incidencia en casos de ambas formas de dengue se encuentra en la región Sur, con apenas 10.731 . El número de muertes por cada 1.000 casos en el período estudiado muestra el año 2015 con la mayor tasa de muertes $(5,81 \%$ ). Conclusiones: El estudio permitió constatar las desigualdades regionales en las políticas de salud pública como: control del vector, eficacia en la notificación y equipo de salud capacitado para la atención.

Descriptores: Dengue, Morbimortalidad, Muertes, DATASUS.

\section{INTRODUÇÃO}

A dengue é uma doença infecciosa febril, com sintomas que incluem febre, geralmente alta $\left(39^{\circ} \mathrm{C} \mathrm{a} 40^{\circ} \mathrm{C}\right)$ de início abrupto, associada a cefaleia, adinamia, mialgias, artralgias, dor retro-orbitária, com presença ou não de exantema. A dengue é classificada em clássica, febre hemorrágica da dengue e dengue com complicações, em que, a primeira é caracterizada pelas manifestaçõs clínicas citadas acima, a segunda por fenomenos hemorrágicos, hepatomegalia e insuficiencia circulatória, e há um achado laboartorial importante como hemoconcentração e trombocitopenia concomitante, e a terceira são alguns casos que não se encaixam na referida classificação. É causada por um vírus transmitido pelo mosquito Aedes aegypt. No Brasil, é uma das doenças mais comuns e atinge as pessoas de todas as classes sociais e em todos estados (SANTOS EA, et al. 2015).

Há um grande impacto mundial causado pela doença. A OMS, Organização Mundial da Saúde, estima que 2,5 bilhões de pessoas estão sob risco de adquirir a dengue e que anualmente cerca de 50 milhões de cidadãos são acometidos pela arbovirose. No Brasil, a primeira epidemia documentada clínica e laboratorialmente foi em 1981-1982, em Boa Vista (RR). Em 1986, houve epidemias no Rio de Janeiro e em algumas capitais nordestinas. A partir disso, vem ocorrendo no Brasil de maneira contínua, geralmente com a introdução de novos sorotipos (NUNES LAS, 2016).

Quando se considera a prevalência de internações por dengue, todos os anos cerca de 500.000 casos de dengue hemorrágica (DH) necessitam desta intervenção. Pelo menos $2,5 \%$ dos casos vão a óbito, mas sugere-se que esta taxa de mortalidade possa ser duas vezes maior, podendo superar os $20 \%$ quando os pacientes não recebem tratamento adequado. Esses índices podem ser reduzidos para menos de 1\%, desde que seja oferecida moderna terapia intensiva de suporte (SINGHI S, et al. 2016).

A partir do aumento populacional e da tendência à urbanização, principalmente em países subdesenvolvidos, agregando-se a aglomeração de casas, muitas vezes sem telhados e com caixas d'água destampadas e saneamento básico deficiente, há um condicionamento para a proliferação e disseminação de vetores e reservatórios de doenças. Os surtos de dengue e a emergência das febres do Chikungunya (CHIKV) e Zika (ZIKAV) transmitidos pelo Aedes aegypti e Albopictus aegypti figuram exemplos dessas enfermidades. O mosquito torna-se capaz de transmitir o vírus após 8 a 12 dias de incubação, desde que ele ingira sangue infectado. Salienta-se que não há transmissão por contato direto com o doente ou com suas secreções (PUSTIGLIONI M, 2016).

Se conhecidos os transmissores de doenças vetoriais, é possível a ajuda e a atuação da sociedade contra a propagação delas. Porém, se a prevenção falha, o tratamento precoce torna-se uma necessidade para que não haja complicações, como hemorragias e/ou mortes (HORTA MAP, et al., 2014).

REAS/EJCH | Vol. Sup. 22 | e753 | DOI: https://doi.org/10.25248/reas.e753.2019 Página 2 de 8 
Uma vez que há exacerbação da doença, considerar que a dengue é um diagnóstico diferencial de Zika e Chikungunya é um passo a mais para evitar seus efeitos graves. Por meio de campanhas de conscientização, estudos acerca da etiopatogenia, propagação e proporções entre dengue clássica e dengue hemorrágica no país podem influenciar esferas do governo e populações sobre a importância de prevenção, diagnóstico e tratamento precoce da dengue. O diagnóstico é feito através da apresentação clínica e exames laboratoriais que incluem: hemograma completo em que pode haver leucopenia, linfocitose com atipia linfocitária e trombocitopenia, coagulograma com aumento dos níveis de protrombina e tromboplastina parcial e trombina, isolamento viral e testes sorológicos como lgM e lgG. Não há tratamento específico com medicação apenas sintomática (DALBEM AG, et al., 2014 e BRASIL, 2016).

Dessa forma, este trabalho tem como objetivo investigar a prevalência de internações por dengue clássica e dengue hemorrágica, no Brasil, entre 2011 e 2015.

\section{MÉTODOS}

$\mathrm{O}$ artigo em questão trata-se de um estudo epidemiológico transversal, descritivo, de abordagem quantitativa, com dados obtidos por meio de banco de dados gerenciado pelo DATASUS, órgão com função de agregar dados estatísticos na área da saúde. Utilizou-se o banco de dados SIH/SUS (Sistema de Informações Hospitalares do Sistema Único de Saúde), onde tal fonte pode ser consultada pelo endereço eletrônico http://www.datasus.gov.com.br.

A seleção de dados realizou-se mediante o recurso TabWin (tecnologia DATASUS), pesquisando em Sistemas de Informação em Saúde (SIS/TabNet) por Epidemiologia e Morbidade no Brasil.

Os estudos englobam indivíduos de ambos os sexos, internados com dengue clássica e dengue hemorrágica, em todas as regiões do Brasil (Norte, Nordeste, Sudeste, Centro-Oeste e Sul). O período de estudo abrangeu os anos de 2011 a 2015 (no período de janeiro de 2011 até dezembro de 2015) e as variáveis coletadas foram as internações por dados sociodemográficos (sexo, faixa etária, cor/raça), distribuição geográfica dos casos, número de internações por ano e taxa de mortalidade.

Os dados foram tabulados e tratados de forma quantitativa, organizados em tabelas e gráficos através do software EXCEL 2013 e submetidos à análise descritiva, apresentados através de distribuição de frequência simples e relativa. Os dados estudados são de domínio público, colhidos a partir do Ministério da Saúde e, portanto, não carecem de avaliação pelo Comitê de Ética em Pesquisa (CEP).

\section{RESULTADOS}

No período de 2011 a 2015 foram registrados 304.444 casos de internações por Dengue Clássica e por Dengue Hemorrágica no Brasil. Pode-se observar que o ano de maior notificação de casos para ambas as formas de dengue foi o de 2011, com total de 80.435 casos. Destes, 76.229 (94,77\%) casos para a Dengue clássica e 4.206 (5,23\%) para a forma Hemorrágica, conforme observado na Tabela 1.

Tabela 1 - Número de internações por Dengue clássica e Dengue hemorrágica entre 2011 e 2015.

\begin{tabular}{cccc}
\hline Ano & Total de internações & DC (N/\%) & DH (N/\%) \\
\hline $\mathbf{2 0 1 1}$ & 80.435 & $76.229(94,77 \%)$ & $4.206(5,23 \%)$ \\
$\mathbf{2 0 1 2}$ & 51.957 & $50.392(96,99 \%)$ & $1.565(3,01 \%)$ \\
$\mathbf{2 0 1 3}$ & 64.850 & $63.188(97,44 \%)$ & $1.662(2,56 \%)$ \\
$\mathbf{2 0 1 4}$ & 36.686 & $35.588(97,01 \%)$ & $1.098(2,99 \%)$ \\
$\mathbf{2 0 1 5}$ & 70.516 & $68.549(97,21 \%)$ & $1.967(2,79 \%)$ \\
TOTAL & 304.444 & $293.946(96,55 \%)$ & $10.498(3,45 \%)$ \\
\hline
\end{tabular}

Fonte: Datasus, 2016 
O total de internações por Dengue Clássica e Dengue hemorrágica no Brasil no período analisado (entre 2011 e 2015) e as características sociodemográficas (sexo, idade, cor/raça) dos indivíduos estudados são descritos na Tabela 2. Neste período, foi observado um maior predomínio do sexo feminino (53,60\%), a faixa etária mais acometida foi de 20 a 39 anos (31,3\%) e a cor/raça mais declarada foi a parda, com 42,7\%.

Tabela 2 - Dados sociodemográficos dos indivíduos internados por Dengue entre 2011 e 2015.

\begin{tabular}{lll}
\hline Variáveis & $\mathbf{N}($ Total $=\mathbf{3 0 4 . 4 4 4 )}$ & $(\%)$ \\
\hline Sexo & 141.233 & 46,40 \\
Masculino & 163.211 & 53,60 \\
Feminino & & \\
Cor/raça & 62.136 & 20,4 \\
Branca & 6.382 & 2,0 \\
Preta & 129.868 & 42,7 \\
Parda & 1.681 & 0,57 \\
Amarela & 357 & 0,13 \\
Indígena & 104.020 & 34,2 \\
Sem informação & & 13,80 \\
Faixa etária (anos) & 42.013 & 20,30 \\
$0-9$ & 61.893 & 31,30 \\
$10-19$ & 95.279 & 21,20 \\
$20-39$ & 64.373 & 13,40 \\
$40-59$ & 40.886 & \\
$>60$ & & \\
\hline
\end{tabular}

Fonte: Datasus, 2016.

Em relação ao número de internações de Dengue clássica e Dengue hemorrágica, por regiões, representados na Tabela 3, pode-se observar que a região Nordeste apresentou o maior número absoluto de casos tanto de DC quanto DH no período estudado, com 128.949 casos no total. A região Sudeste apesar de ser a mais populosa no cenário nacional não apresenta a maior incidência em casos, entretanto possui a maior proporção de casos de $\mathrm{DH}(4,54 \%)$ em relação ao total de casos de dengue. A menor incidência em casos de DC e DH encontra-se na região Sul, com apenas 10.536 e 195, respectivamente.

Tabela 3 - Número de casos de dengue clássica e dengue hemorrágica por regiões de 2011 a 2015.

\begin{tabular}{llll}
\hline REGIÃO & TOTAL & DC (N/\%) & DH (N/\%) \\
\hline Norte & 43.975 & $42.928(97,62 \%)$ & $1.047(2,38 \%)$ \\
Nordeste & 128.949 & $124.873(96,84 \%)$ & $4.076(3,16 \%)$ \\
Sudeste & 76.942 & $73.449(95,46 \%)$ & $3.493(4,54 \%)$ \\
Sul & 10.731 & $10.536(98,18 \%)$ & $195(1,82 \%)$ \\
Centro-oeste & 43.847 & $42.160(3,85 \%)$ & $1.687(3,85 \%)$ \\
\hline
\end{tabular}

Fonte: Datasus, 2016. 
Sobre o número de óbitos registrados para cada 1.000 casos de internações por Dengue hemorrágica e Dengue clássica no período estudado, observou-se que o ano de 2015 apresentou a maior taxa de óbitos, com 5,81\%o (Figura 1).

Figura 1 - Número de óbitos por Dengue por mil habitantes entre 2011 e 2015. Brasil, 2016.
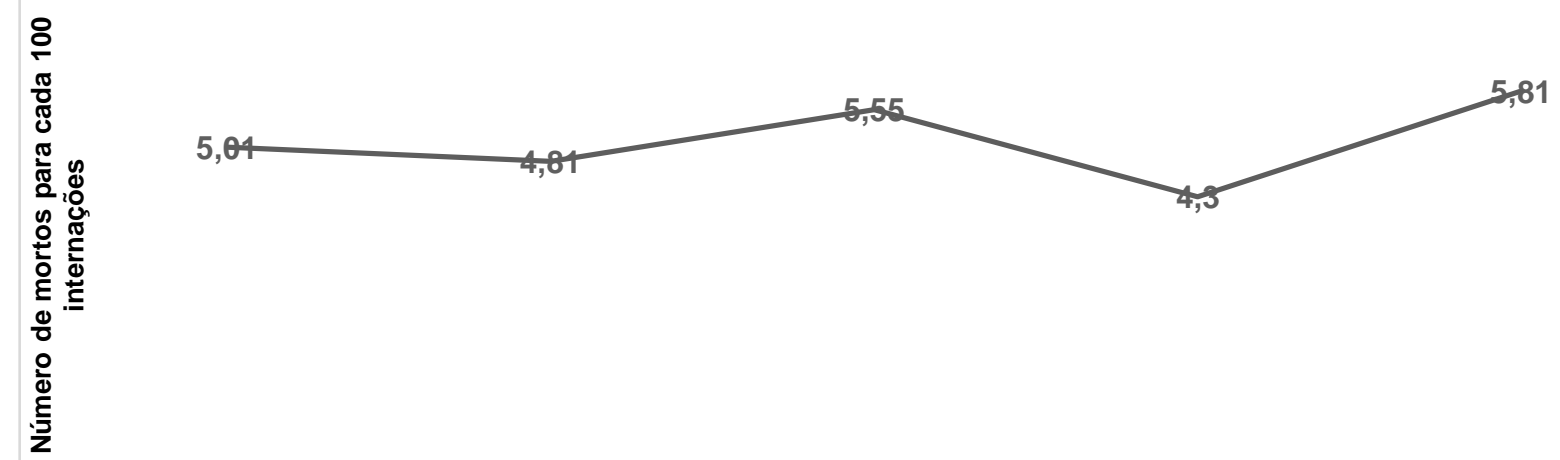

\section{1}

2012

2013

2014

2015

Fonte: Datasus, 2016.

\section{DISCUSSÃO}

Desde os primeiros surtos de dengue no Brasil nas décadas de 80 e 90, tem-se adotado medidas, tanto na implementação de programas de controle ao vetor quanto de conscientização da população, na tentativa de diminuir sua incidência e morbimortalidade. Entretanto, o que se percebe é uma redução temporária e posterior aumento dos índices. Isso tem deixado dúvidas que fazem pensar quais motivos levariam a este quadro atual, sendo necessários esforços no sentido de se realizar estudos populacionais para identificação dos mesmos. (HORTA MAP, et al., 2014).

No presente estudo, no que se refere ao número de internações devido a $\mathrm{DC}$ e $\mathrm{DH}$, no período de 2011 a 2015, observa-se uma grande oscilação nos casos de internação tanto de dengue clássica quanto de hemorrágica entre 2011 e 2012. Tal fato pode ser explicado por vários fatores, como a implantação da Estratégia Global para Prevenção e Controle da Dengue pela OMS, no ano de 2012, que estima diminuir em $50 \%$ a mortalidade e $25 \%$ a morbidade pela doença até o ano de 2020 (WHO, 2016).

Outras medidas que podem ter justificado a redução desse número, principalmente de dengue hemorrágica, são: a sensibilização da população, os investimentos financeiros para o controle do mosquito na ordem de $R \$ 173,2$ milhões para serem repassados a todos os municípios brasileiros, o aumento no efetivo de agentes de controle de endemias garantindo a cobertura das visitas domiciliares, a descentralização das ações voltadas para a dengue, a disseminação de informações relacionadas à dengue na mídia em diversos canais de comunicação, a educação permanente dos profissionais de saúde, e a sensibilização dos gestores da importância de investirem na educação ambiental da população em geral para combater a dengue (ARAÚJO KDS et. al, 2014).

Entretanto, foi possível perceber um aumento do número de internações do ano de 2012 para o de 2013, o que pode ser justificado por outros fatores, como a reintrodução no país do sorotipo DEN-4, em que $60 \%$ dos casos diagnosticados e com o sorotipo isolado em 2013, na cidade de Goiânia, foram de DEN-4 (HORTA MAP, et. al, 2014). Novamente, houve uma queda do número de internações de 2013 para 2014, possivelmente influenciada pelas mesmas medidas anteriormente relatadas (SMS, 2016). 
Surtos de dengue em cidades grandes têm, entre outros fatores da expansão urbana, o número elevado de locais de reprodução do mosquito, altas temperaturas e grande número de pessoas suscetíveis (SMS, 2016). As altas temperaturas, inclusive, podem justificar o pico do número de casos no ano de 2015, considerado o ano mais quente da história do planeta (GRANDELLE R, 2016). A taxa de metabolismo do vetor aumenta nos meses quentes, abreviando seu ciclo evolutivo em até oito dias, ou prolongando-o até 22 dias nos meses frios (CONSOLI RAGB, OLIVEIRA RL, 1994). Também a replicação e maturação do vírus no inseto (período extrínseco) são aceleradas com o aumento da temperatura (FOCKS DA, et. al, 1996)

Entender o perfil das pessoas mais propensas a adquirir a dengue é uma tática importante para prever sua trajetória e então intervir. Deve-se levar em conta que, em relação à dengue, a internação, o afastamento da profissão, a morbidade e a mortalidade, entre outros pontos, geram um custo que, no Brasil, chega a ultrapassar outras doenças virais, como rotavírus e papiloma vírus humano (HPV). Logo, especificar quem são os mais propensos a adoecerem, descobrir o que possuem em comum e intervir, até onde é possível, é uma forma de economizar recursos, possibilitando que o estado possa utilizá-los em outras áreas governamentais (SHEPARD DS, 2011).

Como exposto na Tabela 2, mulheres são as mais afetadas pela doença, indo ao encontro da literatura, que indica mulheres como as mais afetadas tanto em períodos epidêmicos da doença, como em períodos não epidêmicos (LEITE, 2015). Como se sabe, as características clínicas entre dengue e uma gripe comum podem se confundir e o que poderia explicar o fato de mais mulheres serem positivas para dengue do que homens seria o padrão de busca por saúde (DUTRA FCMS, et. al, 2016).

Outro fato é que a faixa etária mais acometida está entre 20 e 39 anos, faixa inclusa na população economicamente ativa do país (entre 10 e 60 anos). Logo, essas pessoas estão propensas a se afastarem do trabalho devido à dengue, principalmente aquelas que podem se expor mais a áreas tendentes ao convívio com o Aedes aegypti (DUTRA FCMS, et. al, 2016).

Quanto à autodeclaração de cor e raça, os dados são pouco confiáveis já que não há informação de $34,2 \%$ dos pesquisados. Contudo, mesmo com $42,7 \%$ se autodeclarando pardo, sabe-se que o vírus da dengue tem suscetibilidade universal e a pesquisa de cor e raça interessa mais por questões sociais (CARROLL ID, et. al, 2007). Sendo assim, cabe salientar ainda o predomínio de pardos em classes mais baixas da sociedade brasileira, o que poderia interferir nos dados (ZZA, 2016).

Quatro sorotipos de dengue circulam nas Américas. Os sorotipos DEN-1, DEN-2 e DEN-3 têm circulado desde 1991 ao mesmo tempo no Brasil (ARAÚJO KDS, et. al, 2014). Segundo a hipótese de infecções sequenciais de Halstead, esta situação representa fator de risco para a ocorrência de febre hemorrágica, devido ao fenômeno de amplificação imune, com presença de anticorpos para sorotipo de dengue em indivíduo infectado por novo sorotipo. Tal fator pode explicar a maior porcentagem de evolução de casos de Dengue clássica para a hemorrágica na região sudeste, pois o Rio de Janeiro é o estado onde há mais tempo circulam os sorotipos DEN-1 e DEN-2 (NOGUEIRA RMR, et. al, 2007), (TEIXEIRA MG, et. al, 2009)

A análise acerca da distribuição das internações nas diferentes regiões do país mostra uma relativa discrepância quando se compara determinadas áreas. A região sudeste, apesar de não ser a com maior prevalência de casos de internação, é a que apresentou mais casos de Dengue clássica que evoluíram para Dengue hemorrágica. $O$ risco de o paciente apresentar dengue hemorrágica é maior quando a segunda infecção é causada pelo DEN-2. A evolução de dengue clássica para dengue hemorrágica ocorre em pequena porcentagem de indivíduos (0,3\% a 4\%) (FERREIRA GLC, 2012).

As baixas notificações vindas da região Sul, em número de casos, de casos graves, incidência e óbitos são explicáveis porque o mosquito Aedes é dependente do fator climático para se desenvolver e não encontra um ambiente favorável na maioria dos estados da região, a população de mosquitos é reduzida em todo país na época de inverno quando as temperaturas caem e as chuvas se tornam escassas na maior parte do Brasil (MARQUES GRAM, et. al, 2008). 
Apesar da importância de a dengue ser uma endemia em ascendência no Brasil e visto que a mesma atinge a população de todos os estados brasileiros, foi verificado que existe apenas um registro patente relacionado a essa arbovirose para o Maranhão, o que pode estar relacionado com a carência de pesquisadores que a estudam (DALBEM AG, et. al, 2014). Em um comparativo, estados brasileiros que mais depositaram patentes sobre a dengue nestes últimos 20 anos foram São Paulo (38), Minas Gerais (31) e Rio de Janeiro (28). No Nordeste, o estado do Ceará é o que mais buscou alternativas contra a dengue, com 5 patentes (ARAÚJO KDS, et. al, 2016). A falta de pesquisadores e pesquisas para controle da endemia na região Nordeste pode ter contribuído para a maior incidência local.

A dengue pode possuir desde formas não aparentes até formas hemorrágicas graves que podem levar ao óbito. Nisso, a Secretaria de Vigilância em Saúde registrou casos de óbitos por dengue no Brasil desde o ano 1980, possuindo o menor número de óbitos em 1996, com apenas um caso (SILVA MM, et al, 2008)

Na Figura 1, nota-se uma variação pequena em relação ao número de óbitos por mil habitantes, uma vez que o Brasil é um país endêmico para a dengue e ainda há um longo trabalho a ser feito por parte da população e governo a fim de prevenir, diagnosticar e tratar precocemente a doença.

Nota-se uma maior variação do número de casos de óbitos por dengue por mil habitantes no período entre 2014 para 2015, visto que há uma variação de 4.3 para 5.81, ou seja, uma variação de $34,8 \%$. Provavelmente, houve essa maior variação devido a uma maior concentração de chuvas em regiões endêmicas, como no sudeste, propiciando assim, um ambiente mais favorável à proliferação do Aedes aegypt e/ou uma maior negligência quanto ao diagnóstico e tratamento precoce da doença, facilitando a evolução para formas mais complicadas, e por consequência, para mortes.

Estudo realizado no sudeste brasileiro investigou a relação entre dengue e mortalidade materna. Observou-se que, no grupo de causas de morte materna, há uma maior prevalência das obstétricas diretas, sendo as síndromes hemorrágicas um dos destaques. Além disso, ao adquirir a dengue durante a gestação pode haver um desenvolvimento ou agravamento de um quadro hemorrágico com variadas consequências, sendo, assim, uma observação de altas taxas de mortalidade nos percentis de maior incidência da dengue (MOTA AKM, et. al, 2012).

O monitoramento dos sorotipos circulantes é crucial visto que a reintrodução de um sorotipo antigo em determinada localidade condiciona a infecção de indivíduos não afetados anteriormente, abrangendo jovens e crianças não nascidos à época, e um novo sorotipo aumenta os riscos de agravamento para dengue grave e síndrome do choque da dengue, para as quais crianças têm 15 vezes mais chances de vir a óbito (VICENTE CR, et al. 2013).

Através do conhecimento dessas características acerca da transmissão, perfil sazonal, prevalência e incidência em determinados grupos sociais e regiões, as causas e agravos da doença, será possível estabelecer um direcionamento de estratégias e medidas de educação e promoção de saúde. Os dados obtidos no presente estudo permitiram evidenciar uma importante desigualdade regional nas políticas de saúde pública que envolvem controle do vetor, eficácia na notificação e equipe de saúde capacitada para o atendimento. Essa desigualdade deve ser considerada para maior eficácia das estratégias de controle, na orientação da população, para o conhecimento dessa endemia que se constitui como problema de saúde coletiva.

\section{CONCLUSÃO}

Conclui-se que houve uma redução das internações por dengue hemorrágica e clássica no período entre 2011 e 2015 no país, havendo oscilações entre os anos. A justificativa da redução diz respeito à melhores e maiores campanhas de conscientização populacional, da implantação da Estratégia Global para Prevenção e do Controle da Dengue pela OMS. Porém, pode-se observar que há ainda discrepância regional acerca das políticas públicas na área da saúde pública como: controle do vetor, eficácia na notificação e equipe de saúde capacitada para o atendimento, dificultando um atendimento e acesso integral ao tratamento das arboviroses. 


\section{AGRADECIMENTO}

Agradecemos às Faculdades Integradas Pitágoras de Montes Claros (FIP-Moc), MG, Brasil e à Prof: Anamaria de Souza Cardoso pela orientação em nosso trabalho.

\section{REFERÊNCIAS}

1. ARAUJO D, CÂNDIDO GA, DANTAS RT. Políticas públicas para a saúde e o papel da atenção básica de saúde no controle e prevenção da dengue no país. Rev Movimentos Sociais e Dinâmicas Espaciais, v. 3, n. 2, p. 247-261, 2014.

2. ARAÚJO KDS, SOUSA SF, SOUSA AAA et al. Dengue: uma prospecção científica e tecnológica no período de 1994 a 2014. Cad Prospecção, v. 9, n. 3, p. 313, 2016.

3. BRASIL. Ministério da Saúde. Secretaria de Vigilância em Saúde. Departamento de Vigilância das Doenças Transmissíveis. Dengue: diagnóstico e manejo clínico: adulto e criança. 5a edição. 2016

4. CARROLL ID, TOOVEY S, GOMPEL AV. Dengue fever and pregnancy - a review and comment. Travel Med Infect Dis, v. 5, n. 3, p. 183-188, 2007.

5. CONSOLI RAGB, OLIVEIRA RL. Principais mosquitos de importância sanitária no Brasil. Ed Fundação Oswaldo Cruz, Rio de Janeiro, 1994.

6. DALBEM AG, HERLING JD, VIEIRA RG et al. Dengue clássica e febre hemorrágica da dengue: etiologia, fisiologia, epidemiologia e fatores de risco. Rev Ciência e Estudos Acadêmicos Med, v. 1, n. 01, 2014.

7. DUTRA FCMS, COSTA LC, SAMPAIO RF. A influência do afastamento do trabalho na percepção de saúde e qualidade de vida de indivíduos adultos. Fisioterapia e Pesquisa, São Paulo, v. 23, n. 1, p. 98-104, Mar. 2016 . Available from <http://www.scielo.br/scielo.php?script=sci_arttext\&pid=S1809-29502016000100098\&lng=en\&nrm=iso>. access on 15 Nov. 2016.

8. FERREIRA GLC. Global dengue epidemiology trends. Rev Inst Med Trop Sao Paulo. São Paulo. 54(18):S5-S6, 2012.

9. FOCKS DA, DANIELS E, HAILE DG, KEESLING JE. A simulation model of the epidemiology of urban dengue fever: literature analysis, model development, preliminary validation and samples of simulations results. Am J Trop Med Hyg, 53:489-506, 1995.

10. GRANDELLE R. 2015 foi $O$ ano mais quente da História, diz Nasa. Disponível em http://oglobo.globo.com/sociedade/sustentabilidade/2015-foi-ano-mais-quente-da-historia-diz-nasa-18512300, acessado em: 19 nov. 2016.

11. HORTA MAP, FERREIRA AP, DE OLIVEIRA RB et al. Os efeitos do crescimento urbano sobre a dengue. Rev Bras em Promoção da Saúde, v. 26, n. 4, p. 539-547, 2014.

12. LEITE PL. Impacto da dengue no Brasil em período epidêmico e não epidêmico: incidência, mortalidade, custo hospitalar e disability adjusted life years (DALY). 2015. 58 [f]. il, Dissertação (Mestrado em Medicina Tropical) - UNB, Brasília, 2015.

13. MARQUES GRAM, SERPA LLN, BRITO M. Aedes aegypti. Laboratório de Culicídeos- SUCEN. Taubaté, p. 105, nov. 2008.

14. MOTA AKM, FILHO ALM, SARACENI V et al. Mortalidade materna e incidência de dengue na Região Sudeste do Brasil: estudo ecológico no período 2001-2005. Cad Saúde Pública, v. 28, n. 6, p. 1057-1066, 2012.

15. NOGUEIRA RMR, DE ARAÚJO JMG, SCHATZMAYR HG. Dengue viruses in Brazil, 1986-2006. Rev Panam Salud Publica, Washington. 22(5):358-63, 2007.

16. NUNES, LAS. Experiência de Macaé/RJ com homeopatia e dengue, 2007-2012. Rev de Homeopatia, v. 79, n. (1/2), p.1-16, 2016.

17. PUSTIGLIONE, M. Medicina do Trabalho e doenças emergentes, reemergentes e negligenciadas: a conduta no caso das febres da dengue, do Chikungunya e do Zika vírus. Rev Bras de Medicina do Trabalho, v. 14, n. 1, p.1-12, 2016.

18. SECRETARIA MUNICIPAL DE SAÚDE DE GOIÂNIA (SMS), Informe Técnico Semanal Dengue, Edição ㄲo 246 - Atualizado em 23/05/2014. Disponível em: http://www.saude.goiania.go.gov.br/docs/divulgacao/Informe\%20dengue\%2023\%200 5\%202014\%20SE\%2020.pdf. Acesso em: 27 de nov 2016.

19. SANTOS EA, MERCES MC, CARVALHO BT. Fatores socioambientais e ocorrência dos casos de dengue em Guanambi-Bahia. Rev de Enfermagem da UFSM, v. 5, n.3, p.486-496, 2015.

20. SHEPARD DS, COUDEVILLE L, HALASA YA et al. Economic Impact of Dengue Illeness in the Americas. Am J Trop Med Hyg, 84(2): 200-207, 2011.

21. SILVA MM, PORTO KRA, ROEL R et al. Informe sobre a dengue em Campo Grande, MS: notificações, causas e consequências para a Saúde Pública. Multitemas, Campo Grande-MS, N.36, P.107-124, 2008.

22. SINGHI S, KISSOON N, BANSAL A. Dengue e dengue hemorrágica: aspectos do manejo na unidade de terapia intensiva. J Ped, Porto Alegre, v. 83, n. 2, supl. p. S22-S35, 2007. Disponível em: <http://www.scielo.br/scielo.php?script=sci_arttext\&pid=S0021-75572007000300004\&lng=en\&nrm=iso>. Acesso em 08 Nov. 2016.

23. TEIXEIRA MG, COSTA MDC, BARRETO F et al. Dengue: twenty-five years since reemergence in Brazil. Cad Saúde Pública.1: S7-S18, 2009.

24. VICENTE CR, LAUAR JC, SANTOS BS et al. Factors related to severe dengue during an epidemic in Vitória, state of Espírito Santo, Brazil, 2011. Rev Soc Bras Med Tropical, v.46, n. 5, p. 629-632, 2013.

25. WHO. Global Strategy for Dengue Prevention and Control: 2012-2020. 2013, Available at: <http://www.who.int/denguecontrol/9789241504034/en/>, acessed on: 19 nov. 2016.

26. ZZA - BRASIL. Ministério do Planejamento, Orçamento e Gestão. Instituto Brasileiro de Geografia e Estatística. Contagem Populacional. Disponível <http://www.sidra.ibge.gov.br/bda/popul/default.asp?t=3\&z=t\&o=22\&u1=1\&u2=1\&u4=1\&u5=1\&u6=1\&u3=34> Acesso em: 27 de nov de 2016 